\title{
1 Associations of Reward Sensitivity with Food Consumption, \\ 2 Activity Pattern, and BMI in Children.
}

3 De Decker, Annelies ${ }^{1}$, Sioen, Isabelle ${ }^{1}$, Verbeken, Sandra ${ }^{2}$, Braet, Caroline $^{2}$, Michels, Nathalie ${ }^{1}$, De

4 Henauw, Stefaan ${ }^{1}$.

$5 \quad{ }^{1}$ Faculty of Medicine and Health Sciences, Department of Public Health, Ghent University, De

6 Pintelaan 185 UZ 4K3, 9000 Gent, Belgium.

7 Annelies.DeDecker@UGent.Be, Isabelle.Sioen@UGent.Be, Nathalie.Michels@UGent.Be,

8 Stefaan.DeHenauw@UGent.Be.

$9 \quad{ }^{2}$ Faculty of Psychology and Educational Sciences, Department of Developmental, Personality and

10 Social Psychology, Ghent University, Henri Dunantlaan 2, 9000 Gent, Belgium.

11 Sandra.Verbeken@UGent.Be, Caroline.Braet@UGent.Be.

13 Corresponding author:

14 Annelies De Decker, Department of Public Health, Ghent University, De Pintelaan 185 UZ 4K3, 159000 Gent, Belgium. E-mail: Annelies.DeDecker@UGent.Be. Telephone: +32 (0)9 3328375. 
 Activity Pattern, and BMI in Children.}

\section{Abstract}

In the current study, the associations of reward sensitivity with weight related behaviors and body mass index were investigated in a general population sample of 443 Flemish children (50.3\% boys) aged 5.5-12 years. Cross-sectional data on palatable food consumption frequency, screen time, physical activity, parental education level and measured length and weight were collected. The Drive subscale of the 'Behavioral Inhibition Scale/Behavioral Activation Scale' was used as a short method to measure reward sensitivity. A significant positive association of reward sensitivity with the fast food and sweet drink consumption frequency was found. Furthermore, a significant positive association of reward sensitivity with the z-score of body mass index was demonstrated, which explained additional variance to the variance explained by palatable food consumption frequency, screen time, physical activity and parental education level. Hence, the assessment of reward sensitivity may have an added value to the assessment of weight-related behavior indicators when evaluating the determinants of overweight in a child. In sum, children high in reward sensitivity might be more attracted to fast food and sweet drinks, and hence, might be more vulnerable to develop unfavorable food habits and overweight. These findings suggest that considering interindividual differences in reward sensitivity is of importance in future childhood obesity prevention campaigns.

Keywords: Reward sensitivity; Palatable food; Child; Body mass index; Overweight.

\section{Introduction $^{1}$}

The prevalence of childhood overweight and obesity has increased dramatically since 1990 (Wang \& Lim, 2012). Since childhood overweight and obesity is associated with multiple adverse health outcomes, the current prevalence is identified as a global public health problem (Baker, Olsen, \& Sorensen, 2007; Deckelbaum \& Williams, 2001; Shrivastava, Shrivastava, \& Ramasamy, 2014). Moreover, overweight and obese youth have an increased risk of maintaining their unfavorable weight status into adulthood (Singh, Mulder, Twisk, van, \& Chinapaw, 2008).

\footnotetext{
${ }^{1}$ Abbreviations: RS, reward sensitivity. BMI, body mass index. PA, physical activity. BIS, behavioral inhibition scales. BAS, behavioral activation scales. PAclub, physical activity in sports clubs. CF, weekly consumption frequency. zBMI, age- and sex-adjusted z-score of body mass index. ISCED, International Standard Classification of Education. PEL, highest parental education level of both parents.
} 
Therefore, it is of the highest importance to prevent childhood overweight and obesity. Unfortunately, current overweight prevention approaches have no or only small effects (Kamath et al., 2008). A better understanding of the determinants of childhood overweight is needed to improve future prevention approaches.

Research has demonstrated positive associations between Body Mass Index (BMI) and the consumption of highly palatable, mostly energy dense foods in children, e.g. fast food (Fraser, Clarke, Cade, \& Edwards, 2012), sugar sweetened beverages (Malik, Pan, Willett, \& Hu, 2013), and artificially sweetened beverages (Sylvetsky, Rother, \& Brown, 2011). For the consumption of sweet food, a significant positive association with BMI was reported in adults, but this association was not demonstrated in children (Te Morenga, Mann, \& Mallard, 2013). Furthermore, positive associations between BMI and screen time (Falbe et al., 2013), and negative associations between BMI and physical activity (PA) have been found in children (Chaput et al., 2014). The current western environment facilitates these unfavorable weight-related behaviors, i.e. a high consumption of widely available palatable foods, prolonged engagement in screen time activities, and sedentary lifestyle combined with low levels of PA (Lowe \& Butryn, 2007). However, not all children exposed to this obesogenic environment display these unfavorable weight-related behaviors and become overweight (Blundell et al., 2005). It has been shown that some individuals are more reactive to the palatable food environment (Paquet et al., 2010), and noteworthy, Forman et al. reported that this reactivity codetermined the effect of the obesity prevention approaches used in their study (Forman et al., 2007). Furthermore, this reactivity to the food environment depends upon individual differences in reward sensitivity (RS) (Paquet et al., 2010).

$\mathrm{RS}$ is the tendency to engage in motivated approach behavior in the presence of environmental cues associated with reward, such as the sight of palatable foods (Carver $\&$ White, 1994). Heightened RS has recently been associated with higher intakes of sugar-sweetened beverages and unhealthy snacks in adolescents (De Cock et al., 2015), and with higher fat intake in adults (Tapper, Baker, Jiga-Boy, Haddock, \& Maio, 2015). Moreover, in normal to overweight adolescents and adults, a positive association was reported between RS and BMI (Davis \& Fox, 2008; Davis et al., 2007; Verbeken, Braet, Lammertyn, Goossens, \& Moens, 2012). Unfortunately, in children, findings are less consistent. One study did not find associations of RS with unhealthy snack consumption and BMI in children (Scholten, Schrijvers, Nederkoorn, Kremers, \& Rodenburg, 2014), while another study reported a positive association between RS and BMI in children, which was mediated by overeating (van den Berg et al., 2011). These inconsistencies might be due to the use of a different RS measure. 
Besides consumption of palatable foods, also screen time (e.g. computer games) and PA (e.g. endurance running) were reported to have rewarding potential (Buckley, Cohen, Kramer, McAuley, \& Mullen, 2014; Garland et al., 2011). This might implicate that RS also plays a role in those weight-related behaviors (Buckley et al., 2014). Nevertheless, literature on the association of RS with PA and screen time is to our knowledge absent in children and adolescents. In adults, one study reported no relation between RS and PA (Finlayson, Cecil, Higgs, Hill, \& Hetherington, 2012), whereas another study reported more PA in individuals with higher RS (Voigt et al., 2009).

Since previous research suggested that knowledge on the association of RS with weightrelated behaviors and $\mathrm{BMI}$ is imperative for the development of effective prevention strategies, the current study aimed to investigate these associations in a large general population sample of children aged 5.5-12 years. Therefore, consumption frequencies of different types of palatable food, screen time, PA, and BMI calculated upon measured weight and height were used. In accordance to the studies in adolescents (De Cock et al., 2015; Verbeken et al., 2012), the current study used the Drive subscale of the 'Behavioral Inhibition/ Behavioral Activation Scales' (BIS/BAS) as a measure of RS, which is conceptualized as the motivation to approach potentially pleasurable activities (Carver \& White, 1994). Important advantages of the Drive subscale are that (a) it was validated in neuro-imaging research (Beaver et al., 2006), (b) it is a short 4-item scale, easily and practically applicable in epidemiological research, obesity prevention interventions and clinical practice, and (c) it does not only measures reactivity to food, but to all kinds of reward, such that it has the potential to be associated with food consumption as well as screen time and PA.

A positive association between RS and the consumption of high-fat fast food, sweet food, and sugared and artificially sweetened beverages was hypothesized. Additionally, the relation of RS with screen time and PA was explored. Further, it was hypothesized that RS was positively associated with BMI and explained additional variance of BMI to the assessment of known predictors of BMI (i.e. palatable food consumption, screen time, PA and parental education level).

\section{Method}

Study participants

Participants were Dutch-speaking Belgian children aged 5.5-12 years, recruited by random cluster design for the longitudinal Children's Body Composition and Stress (ChiBS) study (Michels et al., 2012) that took place between 2010 and 2012. Children (in most cases accompanied by minimum one parent) attended the survey centre at a prefixed appointment, during which the 
anthropometric measurements of the child were conducted and questionnaires were filled in by the parent. If the parent could not accompany the child, the parents were asked to fill in the questionnaires at home.

The 455 children that participated in the ChiBS study wave of 2011 were included in the current study. Of the 455 children, twelve children were excluded from the analyses (nine had missing RS-data; three children reached the criteria for obesity, see discussion for argumentation on exclusion of children with obesity). As such, the total study sample consisted of 443 children. A post hoc power calculation was performed based on a sample size of 443 children and the mean of the two squared correlation coefficients (i.e. 0.02) reported in the study of van den Berg et al. that demonstrated a significant relation between scores on two RS measures and BMI in children (van den Berg et al., 2011). This revealed a power of 0.79 to detect a true effect between RS and BMI in the current study.

The ChiBS study was conducted according to the guidelines laid down in the Declaration of Helsinki and was approved by the Ethics Committee of Ghent University Hospital. Written informed consent was obtained from all parents and the children gave verbal assent.

\section{Measures}

$\underline{\mathrm{RS}}$. The BAS scale of the BIS/BAS scale consists of three subscales, namely the Drive, Reward Responsiveness, and Fun Seeking subscale (Carver \& White, 1994). The Drive subscale was designed to reflect strong pursuit of appetitive goals and consists of four items which all need to be scored on a 4-point Likert scale (1=not true, $2=$ somewhat true, $3=$ true, $4=$ very true; items are (a) when your child wants something, he/she usually goes all the way to get it, (b) your child does everything to get the things that he/she wants, (c) when your child sees an opportunity to get something that he/she wants, he/she goes for it right away, (d) nobody can stop your child when he/she wants something). Of the three BAS subscales, it has the highest internal consistency (De Cock et al., 2015; Jorm et al., 1999) and the strongest relations with palatable food intake in adolescents (De Cock et al., 2015). Furthermore, the Drive subscale is strongly associated with neural responses to appetizing food-reward cues in the brain reward circuitry, and this association is stronger than the associations between these neural responses and the other BAS subscales (Beaver et al., 2006). Therefore, the term RS refers to the sum of the four items of the Drive subscale. Because the youngest children of the cohort were too young to answer the questionnaire themselves, parents answered a Dutch parent version of the BIS/BAS scale (Vervoort et al., 2015). The Cronbach alpha coefficient of RS in the current study (0.85) was comparable to the alpha 
reported by Vervoort et al. (i.e. 0.85) in children and adolescents aged 2-18 years (Vervoort et al., 2015).

Food indices. Parents completed the Children's Eating Habits Questionnaire - Food Frequency Questionnaire to report the child's usual weekly consumption frequency $(\mathrm{CF})$, thereby considering the preceding 4 weeks. The questionnaire consists of 43 food items/categories and was developed and validated within the EU FP6 IDEFICS project (Huybrechts et al., 2011; Lanfer et al., 2011). For each item, the following response options were used (the assigned score is indicated in brackets): 'never/less than once a week' (value 0), 'one to three times a week' (value 2), 'four to six times a week' (value 5), 'one time a day' (value 7), 'two times a day' (value 14), 'three times a day' (value 21), 'four or more times a day' (value 30), or 'I have no idea' (missing). Based on this questionnaire, three food indices were calculated by summing up the weekly CF's of related food items/categories: (a) Fast food CF, contains all fast food and combined sauces categories; (b) Sweet food CF, contains all sweet food categories; (c) Sweet drink CF, contains all sweet tasting drink categories (Table 1).

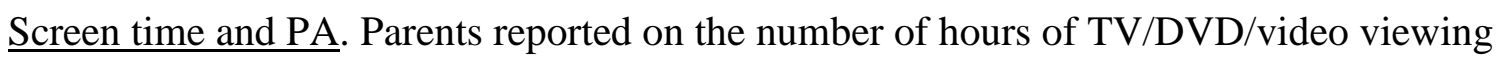
and computer/games-console use both for typical weekdays and weekend days. Response categories included: 'not at all' (value zero), '<0.5 hours a day' (value 0.25 ), '<1 hours a day' (value 0.75 ), 'between 1 and $<2$ hours a day' (value 1.5), 'between 2 and $<3$ hours a day' (value 2.5), '>3 hours a day' (value 4). Children's weekly TV/DVD/video viewing (5 times week and 2 times weekend viewing) and computer/games-console use (5 times week and 2 times weekend use) were summed to obtain the hours of screen time per week (Olafsdottir et al., 2014).

Parental report on "How much hours and minutes does he/she spend doing sport in a sports club per week" (no response categories) was used as a proxy measure for moderate to vigorous PA, further referred to as PA at sports clubs (PAclub).

BMI. Height $(\mathrm{m})$ and body mass $(\mathrm{kg})$ were measured. Children were not allowed to eat or drink during 2 hours preceding the weighing. Upon weight and length data, BMI $\left(\mathrm{kg} / \mathrm{m}^{2}\right)$ was calculated, and the standard deviation score of BMI (zBMI) was computed to adjust for age- and sex using xILMS (i.e. an excel add-in for using growth reference data in the LMS format; abbreviation LMS refers to smooth curve-L, mean-M and coefficient of variation-S) with Flemish growth reference data of 2004 (Cole, Freeman, \& Preece, 1998; Roelants, Hauspie, \& Hoppenbrouwers, 2009). According to the cut-offs of the International Obesity Task Force (Cole \& Lobstein, 2012), children with BMI z-scores of $\geq 2.29$ for boys and $\geq 2.19$ for girls (equivalent of BMI 30 at age 18) were classified as obese, and excluded from further analyses. 

categorized according to the International Standard Classification of Education (ISCED)

189 190 191 192 193 194 195 196 197 198 199 200 201 202 203 204 205 206 207 208 209 210 211 212 213 214 215 216 217 218 219 (UNESCO, 1997). Because of low numbers of participants in category zero to four, the ISCEDcategories were aggregated into two levels (ISCED level 0 - 4 = low PEL, value zero; ISCED level 5-6 = high PEL, value one).

\section{Statistical analyses}

Analyses were performed using PASW Statistical Program version 20.0 (SPSS, IBM, IL, USA). The two-sided level of significance was set at $\mathrm{p}<0.05$. Missing values were not estimated since most missing values were the consequence of questionnaires that were not filled in due to time constraints. Histograms and boxplots were drawn to identify outliers and non-normal distributions. Based on visual inspection, it was decided to exclude five extreme outliers of the sweet drink CF and two of the sweet food CF from further analyses. The fast food, sweet food, and sweet drink CF, and PAclub were found to be non-normally distributed. To use the food indices as dependent variables in regressions, correlations, and t-tests, value one was added to the scores on the single food items of the fast food, sweet food, and sweet drink CF, resulting in a food frequency range of $[1 ; 31]$ instead of $[0 ; 30]$. Then, the fast food $\mathrm{CF}$, sweet food $\mathrm{CF}$ and sweet drink CF sum scores were calculated again, and the natural logarithms $(\ln )$ of all food indices were computed, which approached the normal distribution. For PAclub, transformations did not change the distribution towards normality. To use PAclub as dependent variable in regressions, it was dichotomized (zero to two hours per week = low PAclub, value zero; more than two hours per week= high PAclub, value one).

Explorative unadjusted Pearson correlations (exception: Spearman correlation for PAclub) and unpaired t-tests (exception: Mann-Whitney $U$ test for PAclub) were conducted to find out if age, sex and PEL had to be included as covariates when regressing weight-related behaviors on RS. They were only included as covariates in regression models if (trend) significant associations were present between age, sex or PEL and (a) the predictor, and (b) the dependent variable.

To investigate the research hypothesis that RS was positively associated with the three food indices, three linear regression models were conducted with RS as predictor and fast food CF, sweet food $\mathrm{CF}$ and sweet drink $\mathrm{CF}$ as dependent variables. To explore if RS was associated with screen time and PAclub, a linear and a logistic regression were conducted respectively with RS as predictor and screen time and PA club as dependent variables. 
To investigate if RS was positively associated with zBMI and if it explained additional variance to the assessment of known predictors, a hierarchical linear regression model with zBMI as dependent variable was conducted. This analysis was conducted on a subsample of the total study sample, for which all predictors included in the model were reported. In step 1 of the hierarchical linear regression model, the three food indices, screen time, PAclub and PEL were added as

\section{Association of $R S$ with food indices, screen time and PAclub}

Explorative analyses. Correlations and comparisons were performed to find out which variables should be included as covariates in the regression models with RS as predictor and weight-related behaviors as dependent variables (Table 3). Based on these results, age was included as covariate in all five regression models with RS as predictor and the weight-related behaviors as dependent variables because (a) a trend significant correlation between RS and age was present, and (b) age was significantly related to screen time and PAclub, and trend significant to the fast food 
and sweet food CF. Sex was only included as covariate in the regression model with screen time as dependent variable, since trend significant sex differences were only present on RS and screen time. PEL was not included as covariate in the regression models: although the CF of fast food and sweet drink were significantly higher in low PEL (mean fast food CF low PEL=7.61, high PEL=5.57 times a week; mean sweet drink CF low PEL=11.39, high PEL=8.69 times a week), no association was found between PEL and RS.

Regression analyses adjusted for covariates. Table 4 shows the results of the five regressions. RS was significantly and positively related to the fast food $\mathrm{CF}$ and sweet drink $\mathrm{CF}$, but not to the sweet food CF, screen time, and PAclub. The models predict that children aged 8.86 years (i.e. mean age) at percentile 10 versus 90 of RS consume fast food on average 4.89 versus 5.85 times a week respectively, and consume sweet drinks on average 6.95 versus 8.42 times a week, respectively.

Association of $R S$ with zBMI

RS was significantly and positively associated with zBMI (Table 5). RS significantly explained an extra $2 \%$ of the variance in $\mathrm{zBMI}$ to the variance explained by the weight-related behaviors and PEL. Tolerance values to check multicollinearity were all above 0.8 in both steps of the model. The model predicts that children at percentile 90 of RS have on average a 0.34 units higher zBMI than children at percentile 10 of RS.

\section{Discussion}

The present study investigated the associations of the scores on a short RS questionnaire, namely the Drive subscale of the BIS/BAS scale (Carver \& White, 1994), with weight related behaviors and zBMI in a general population sample of children aged 5.5-12 years.

The results confirmed that children with higher RS may consume more frequently fast food and sweet drinks. Similar findings were recently found in adolescents aged 14-16 years (De Cock et al., 2015). The current study findings suggest that even in children, whose access to food is strongly determined by others (e.g. parents, teachers), the individual characteristic RS may play a role in palatable food consumption. Children high in RS might be more easily tempted by palatable fast food and sweet drink cues, and more motivated to consume them. This might shape unfavorable food habits that continue during life. 
The research hypothesis that RS was positively associated with the sweet food CF could not be confirmed. Nevertheless, in line with other studies, the sweet food CF was not related to PEL, whereas the fast food and sweet drink CF were related to PEL (Elinder, Heinemans, Zeebari, \& Patterson, 2014). Possibly, the fast food and sweet drink CF might be determined by different parameters (PEL, RS) than the sweet food CF, which might be determined by habits in Flemish primary schools (eating biscuits and/or chocolate bars as snacks during school breaks is common).

The present study found no associations between RS and the potentially rewarding behaviors "screen time" and "physical activity in sports clubs". Previous research on this associations in primary school children is to our knowledge absent. Possibly, RS does not influence these weight-related behaviors. Nonetheless, the lack of a relation between RS and screen time might also be due to high parental control or restriction over screen time in this age group. Additionally, screen time was measured in the current study by the sum of 'hours of television viewing' and 'hours in front of a computer/game console', but only the hours of engagement in rewarding computer games might be related to RS. Future research is therefore needed to replicate these findings with refined measures of screen time, thereby differentiating between television and gaming. The lack of a relation between RS and PAclub was in line with results of a systematic review on children and adolescents, which concluded that PAclub was more consistently related to environmental characteristics than to interpersonal factors (de Vet, de Ridder, \& de Wit, 2011). Indeed, whether children like sporting in a sports club or not, the hours of PAclub of primary school children is dependent on the permission and logistic support of their parents and on nearby sports club facilities. Further, future research in children might explore the relationship between RS and objectively measured physical activity (e.g. with accelerometers), assessing the overall level of PA during daytime.

In the current study, only a trend significant positive association between the fast food CF and zBMI was present. No associations of the sweet drink CF, screen time, and PAclub with zBMI were found. Recent reviews indicated that only some studies reported significant relations between these parameters and BMI (Malik et al., 2013; Must, Barish, \& Bandini, 2009). Probably, differences in methodology can explain the different findings. Further, a negative relation between sweet food CF and zBMI was found, which is in conflict with a meta-analysis that reported no significant relationship between sweet food and BMI in children (Te Morenga et al., 2013). This negative association might be due to the assessment of consumption frequencies without inquiring portion sizes. Portion sizes of sweet food CF might vary substantially across children (e.g. one versus three cookies per consumption). Hence, future research should include more detailed assessment of dietary habits, identifying not only frequency but also portion size. 
Finally, the current study demonstrated a positive association between RS, measured by the

322 Drive subscale of the BIS/BAS scale, and zBMI in normal to overweight children. This finding was 323 already reported in adolescents with the same RS questionnaire (Verbeken et al., 2012) and in 324 children with a different RS questionnaire (van den Berg et al., 2011). Another study in children 325 that used a behavioral task as RS measure did not find this association (Scholten et al., 2014). 326 Moreover, RS assessment explained additional variance of zBMI to the variance explained by food 327 consumption, activity pattern, and PEL. Therefore, the assessment of RS with this very short 328 questionnaire might have an added value in public health and pediatrics.

329 The positive association between RS and zBMI was found in a population of children 330 without obesity. Children with obesity were excluded from the analyses because (a) the focus of this 331 study is on obesity prevention, (b) the obesity rate in the current sample was too low to accurately 332 investigate the relation between RS and zBMI in obese children, and (c) most importantly, RS was 333 only positively associated with BMI in adolescents (Verbeken et al., 2012) and adults (Davis \& 334 Fox, 2008) without obesity, but negatively associated with BMI in the obese population in both 335 studies. This inverted relation is probably due to changes in brain reward processes over the course 336 of obesity development (Kessler, Zald, Ansari, Li, \& Cowan, 2014).

The positive associations of RS with fast food CF, sweet drink CF and zBMI reported in this paper are relevant for future prevention strategies, certainly if future longitudinal studies can confirm an increased obesity risk in high RS children. Such associations can offer an explanatory 340 framework for parents and health care workers on why some children are more tempted by palatable food compared to other children. Further, specifically targeting children high in RS, which are assumed to be more vulnerable to the obesogenic environment, may improve the effectiveness of obesity prevention interventions. In fact, there is already some evidence in adults as to which elements to include in prevention interventions tailored to this RS feature. Three such elements are worth briefly describing in the context of this paper. First, messages that are framed in terms of the benefits of adopting the recommendation (i.e. gain-frame) rather than the disadvantages and costs of not adopting a recommendation are more effective in high RS individuals (Covey, 2014). Second, the study of Forman et al. (2007) compared two methods designed to help individuals manage palatable food cravings such that they do not lead to palatable food consumption: (a) 'control-based strategies', e.g. removing palatable foods from the direct home or work environment, restructuring thoughts that permit eating palatable food, and refocusing strategies designed to turn attention away from food related stimuli towards non-food related stimuli; (b) acceptance-based strategies, e.g. awareness and acceptance of the feelings of food cravings without trying to suppress or eliminate them and without taking actions in order to consume the desired food. The method with acceptance- 
based strategies decreased the consumption of palatable foods in participants with high RS specific to food, but increased food cravings in participants with low RS specific to food. Hence, interventions using these acceptance-based strategies are useful only in high RS individuals. Third, self-regulatory skills were found to moderate the relation between RS and BMI in adults (Lawrence, Hinton, Parkinson, \& Lawrence, 2012). Therefore, the training of self-regulatory skills (Verbeken, Braet, Goossens, \& van der Oord, 2013) might be effective to reduce palatable food consumption in high RS individuals. Future research should clarify if also in children, these three intervention techniques can be successfully applied.

The limitations of the current study include its cross-sectional design. Future longitudinal research to confirm causality is needed. Further, although BMI is a frequently used measure of adiposity, better measures exist (e.g. densitometry). Next, children with overweight and obesity, as well as families with lower levels of parental education were relatively underrepresented in the current study. Therefore, future research in a more representative sample is recommended. Additionally, RS and all weight-related behaviors were based on subjective questionnaires. The construct of RS could be confounded by parenting style, and the relation of RS and palatable food consumption in children could be confounded by food provision patterns of parents, which were not taken into account in the current study. Further, the PAclub measure did not include PA of the child outside of sports clubs. The number of missing values on weight-related variables was high due to time constraints of parents. However, no differences were found in RS and zBMI between the total sample and the subsample. Because inclusion of portion size assessment in a cohort study is a high burden for participants and would result in a reduced sample size, the food indices were only based on consumption frequency assessments. Therefore, associations between the three food indices and zBMI should be interpreted with caution. Unless the mentioned limitations, relationships between the weight-related parameters screen time, the fast food $\mathrm{CF}$, sweet food $\mathrm{CF}$, sweet drink $\mathrm{CF}$, and PEL were as expected based on the literature (Fernandez-Alvira et al., 2015; Pearson \& Biddle, 2011; Tandon et al., 2012). Further, the strengths of the current study include the large general community sample of primary school children, the use of a simple and short RS questionnaire, the consideration of multiple weight-related behaviors, and the objective measurement of weight and length.

\section{Conclusion}

Overall, the results of the current study suggest that children high in RS are more easily tempted by palatable fast food and sweet drink cues, which might lead to unhealthy food habits. 
389 Further, children high in RS might be more prone to develop overweight. These findings suggest

390 that considering inter-individual differences in RS can be of importance in future childhood obesity 391 prevention campaigns. Future longitudinal research is warranted to verify that RS is a risk factor of 392 unfavorable food habits and overweight in children.

393

394 Acknowledgments

395

396 The authors want to thank the participating children and their parents for their voluntary

397 participation.

398

399 Financial support

400

401 This work was supported by the research council of Ghent University, the Institute for the

402 Promotion of Innovation through Science and Technology in Flanders (grant number SBO-120054),

403 and the Research Foundation - Flanders (I.S., grant number 1.2.683.14.N.00). These funders had no

404 role in the design, data collection, analyses or writing of this article, or the decision to submit this

405 article for publication. Further, this research received no specific grant from any commercial

406 funding agency. 


\section{References}

Aloe, A. M. (2014). An Empirical Investigation of Partial Effect Sizes in Meta-Analysis of Correlational Data. Journal of General Psychology, 141(1), 47-64. doi:10.1080/00221309.2013.853021

Baker, J. L., Olsen, L. W., \& Sorensen, T. I. (2007). Childhood body-mass index and the risk of coronary heart disease in adulthood. New England Journal of Medicine, 357(23), 23292337.

Beaver, J. D., Lawrence, A. D., Van Ditzhuijzen, J., Davis, M. H., Woods, A., \& Calder, A. J. (2006). Individual differences in reward drive predict neural responses to images of food. Journal of Neuroscience, 26(19), 5160-5166. doi:10.1523/jneurosci.0350-06.2006

Blundell, J. E., Stubbs, R. J., Golding, C., Croden, F., Alam, R., Whybrow, S. et al. (2005). Resistance and susceptibility to weight gain: individual variability in response to a high-fat diet. Physiology \& Behavior, 86(5), 614-622.

Buckley, J., Cohen, J. D., Kramer, A. F., McAuley, E., \& Mullen, S. P. (2014). Cognitive control in the self-regulation of physical activity and sedentary behavior. Frontiers in Human Neuroscience, 8. doi:10.3389/Mhum.2014.00747

Carver, C. S., \& White, T. L. (1994). Behavioral-Inhibition, Behavioral Activation, and Affective Responses to Impending Reward and Punishment - the Bis Bas Scales. Journal of Personality and Social Psychology, 67(2), 319-333.

Chaput, J. P., Leduc, G., Boyer, C., Belanger, P., LeBlanc, A. G., Borghese, M. M., \& Tremblay, M. S. (2014). Objectively measured physical activity, sedentary time and sleep duration: independent and combined associations with adiposity in canadian children. Nutrition \& Diabetes, 4, 5. doi:10.1038/nutd.2014.14

Cohen, J. (1992). A POWER PRIMER. Psychological Bulletin, 112(1), 155-159. doi:10.1037/00332909.112.1.155

Cole, T. J., Freeman, J. V., \& Preece, M. A. (1998). British 1990 growth reference centiles for weight, height, body mass index and head circumference fitted by maximum penalized likelihood. Statistics in Medicine, 17(4), 407-429. doi:10.1002/(sici)10970258(19980228)17:4<407::aid-sim742>3.0.co;2-1

Cole, T. J., \& Lobstein, T. (2012). Extended international (IOTF) body mass index cut-offs for thinness, overweight and obesity. Pediatric Obesity, 7(4), 284-294. doi:10.1111/j.20476310.2012.000064.x

Covey, J. (2014). The Role of Dispositional Factors in Moderating Message Framing Effects. Health Psychology, 33(1), 52-65. doi:10.1037/a0029305 
Davis, C., \& Fox, J. (2008). Sensitivity to reward and body mass index (BMI): evidence for a nonlinear relationship. Appetite., 50(1), 43-49.

Davis, C., Patte, K., Levitan, R., Reid, C., Tweed, S., \& Curtis, C. (2007). From motivation to behaviour: a model of reward sensitivity, overeating, and food preferences in the risk profile for obesity. Appetite., 48(1), 12-19.

De Cock, N., Van Lippevelde, W., Vervoort, L., Vangeel, J., Maes, L., Eggermont, S., et al. (2015). Sensitivity to reward is associated with snack and sugar-sweetened beverage consumption in adolescents. European journal of nutrition. doi:10.1007/s00394-015-0981-3

de Vet, E., de Ridder, D. T. D., \& de Wit, J. B. F. (2011). Environmental correlates of physical activity and dietary behaviours among young people: a systematic review of reviews. Obesity Reviews, 12(501), e130-e142. doi:10.1111/j.1467-789X.2010.00784.X

Deckelbaum, R. J., \& Williams, C. L. (2001). Childhood obesity: the health issue. Obesity Research, 9(Suppl. 4), S239-S243.

Elinder, L. S., Heinemans, N., Zeebari, Z., \& Patterson, E. (2014). Longitudinal changes in health behaviours and body weight among Swedish school children - associations with age, gender and parental education - the SCIP school cohort. Bmc Public Health, 14, 9. doi:10.1186/1471-2458-14-640

Falbe, J., Rosner, B., Willett, W. C., Sonneville, K. R., Hu, F. B., \& Field, A. E. (2013). Adiposity and Different Types of Screen Time. Pediatrics, 132(6), E1497-E1505. doi:10.1542/peds.2013-0887

Fernandez-Alvira, J. M., Bornhorst, C., Bammann, K., Gwozdz, W., Krogh, V., Hebestreit, A., et al. (2015). Prospective associations between socio-economic status and dietary patterns in European children: the Identification and Prevention of Dietary- and Lifestyle-induced Health Effects in Children and Infants (IDEFICS) Study. British journal of nutrition, 113(3), 517-525. doi:10.1017/s0007114514003663

Finlayson, G., Cecil, J., Higgs, S., Hill, A., \& Hetherington, M. (2012). Susceptibility to weight gain. Eating behaviour traits and physical activity as predictors of weight gain during the first year of university. Appetite, 58(3), 1091-1098. doi:10.1016/j.appet.2012.03.003

Forman, E. M., Hoffman, K. L., McGrath, K. B., Herbert, J. D., Brandsma, L. L., \& Lowe, M. R. (2007). A comparison of acceptance- and control-based strategies for coping with food cravings: an analog study. Behaviour Research and Therapy, 45(10), 2372-2386.

Fraser, L. K., Clarke, G. P., Cade, J. E., \& Edwards, K. L. (2012). Fast Food and Obesity A Spatial Analysis in a Large United Kingdom Population of Children Aged 13-15. American Journal of Preventive Medicine, 42(5), E77-E85. doi:10.1016/j.amepre.2012.02.007 
Garland, T., Jr., Schutz, H., Chappell, M. A., Keeney, B. K., Meek, T. H., Copes, L. E., et al. (2011). The biological control of voluntary exercise, spontaneous physical activity and daily energy expenditure in relation to obesity: human and rodent perspectives. Journal of Experimental Biology, 214(2), 206-229. doi:10.1242/jeb.048397

Huybrechts, I., Boernhorst, C., Pala, V., Moreno, L. A., Barba, G., Lissner, L., et al. (2011). Evaluation of the Children's Eating Habits Questionnaire used in the IDEFICS study by relating urinary calcium and potassium to milk consumption frequencies among European children. International Journal of Obesity, 35, S69-S78. doi:10.1038/ijo.2011.37

Jorm, A. F., Christensen, H., Henderson, A. S., Jacomb, P. A., Korten, A. E., \& Rodgers, B. (1999). Using the BIS/BAS scales to measure behavioural inhibition and behavioural activation: Factor structure, validity and norms in a large community sample. Personality and Individual Differences, 26(1), 49-58.

Kamath, C. C., Vickers, K. S., Ehrlich, A., McGovern, L., Johnson, J., Singhal, V., et al. (2008). Behavioral Interventions to Prevent Childhood Obesity: A Systematic Review and Metaanalyses of Randomized Trials. Journal of Clinical Endocrinology \& Metabolism, 93(12), 4606-4615. doi:10.1210/jc.2006-2411

Kessler, R. M., Zald, D. H., Ansari, M. S., Li, R., \& Cowan, R. L. (2014). Changes in Dopamine Release and Dopamine D2/3 Receptor Levels with the Development of Mild Obesity. Synapse, 68(7), 317-320. doi:10.1002/syn.21738

Lanfer, A., Hebestreit, A., Ahrens, W., Krogh, V., Sieri, S., Lissner, L., et al. (2011). Reproducibility of food consumption frequencies derived from the Children's Eating Habits Questionnaire used in the IDEFICS study. International Journal of Obesity, 35, S61-S68. doi:10.1038/ijo.2011.36

Lawrence, N. S., Hinton, E. C., Parkinson, J. A., \& Lawrence, A. D. (2012). Nucleus accumbens response to food cues predicts subsequent snack consumption in women and increased body mass index in those with reduced self-control. Neuroimage, 63(1), 415-422.

Lowe, M. R., \& Butryn, M. L. (2007). Hedonic hunger: A new dimension of appetite? Physiology \& Behavior, 91(4), 432-439.

Malik, V. S., Pan, A., Willett, W. C., \& Hu, F. B. (2013). Sugar-sweetened beverages and weight gain in children and adults: a systematic review and meta-analysis. American Journal of Clinical Nutrition, 98(4), 1084-1102. doi:10.3945/ajcn.113.058362

Michels, N., Vanaelst, B., Vyncke, K., Sioen, I., Huybrechts, I., De Vriendt, T., \& De Henauw, S. (2012). Children's Body composition and Stress - the ChiBS study: aims, design, methods, population and participation characteristics. Archives of Public Health, 70(1), 17. 
Must, A., Barish, E. E., \& Bandini, L. G. (2009). Modifiable risk factors in relation to changes in BMI and fatness: what have we learned from prospective studies of school-aged children? International Journal of Obesity, 33(7), 705-715. doi:10.1038/ijo.2009.60

Olafsdottir, S., Berg, C., Eiben, G., Lanfer, A., Reisch, L., Ahrens, W., et al. (2014). Young children's screen activities, sweet drink consumption and anthropometry: results from a prospective European study. European Journal of Clinical Nutrition, 68(2), 223-228.

Paquet, C., Daniel, M., Knauper, B., Gauvin, L., Kestens, Y., \& Dube, L. (2010). Interactive effects of reward sensitivity and residential fast-food restaurant exposure on fast-food consumption. American Journal of Clinical Nutrition, 91(3), 771-776.

Pearson, N., \& Biddle, S. J. H. (2011). Sedentary Behavior and Dietary Intake in Children, Adolescents, and Adults A Systematic Review. American Journal of Preventive Medicine, 41(2), 178-188. doi:10.1016/j.amepre.2011.05.002

Roelants, M., Hauspie, R., \& Hoppenbrouwers, K. (2009). References for growth and pubertal development from birth to 21 years in Flanders, Belgium. Annals of Human Biology, 36(6), 680-694.

Scholten, E. W., Schrijvers, C. T., Nederkoorn, C., Kremers, S. P., \& Rodenburg, G. (2014). Relationship between impulsivity, snack consumption and children's weight. Plos One, 9(2), e88851.

Shrivastava, S. R., Shrivastava, P. S., \& Ramasamy, J. (2014). Childhood obesity: a determinant of adolescent and adult hypertension. International Journal of Preventive Medicine, 5(Suppl 1), S71-S72.

Singh, A. S., Mulder, C., Twisk, J. W., van, M. W., \& Chinapaw, M. J. (2008). Tracking of childhood overweight into adulthood: a systematic review of the literature. Obesity Reviews, $9(5), 474-488$.

Sylvetsky, A., Rother, K. I., \& Brown, R. (2011). Artificial Sweetener Use Among Children: Epidemiology, Recommendations, Metabolic Outcomes, and Future Directions. Pediatric Clinics of North America, 58(6), 1467-1480. doi:10.1016/j.pcl.2011.09.007

Tandon, P. S., Zhou, C., Sallis, J. F., Cain, K. L., Frank, L. D., \& Saelens, B. E. (2012). Home environment relationships with children's physical activity, sedentary time, and screen time by socioeconomic status. International Journal of Behavioral Nutrition and Physical Activity, 9, 9. doi:10.1186/1479-5868-9-88

Tapper, K., Baker, L., Jiga-Boy, G., Haddock, G., \& Maio, G. R. (2015). Sensitivity to reward and punishment: Associations with diet, alcohol consumption and smoking. Personality and Individual Differences, 72, 79-84. doi:10.1016/j.paid.2014.08.025 
Te Morenga, L. A., Mann, J., \& Mallard, S. (2013). Dietary sugars and body weight: systematic review and meta-analyses of randomised controlled trials. BMJ-British Medical Journal, 346. doi:10.1136/bmj.e7492

UNESCO. (1997). International standard classification of education.

van den Berg, L., Pieterse, K., Malik, J. A., Luman, M., van Dijk, K. W., Oosterlaan, J., \& Delemarre-van de Waal, H. A. (2011). Association between impulsivity, reward responsiveness and body mass index in children. International Journal of Obesity, 35(10), 1301-1307.

Verbeken, S., Braet, C., Goossens, L., \& van der Oord, S. (2013). Executive function training with game elements for obese children: A novel treatment to enhance self-regulatory abilities for weight-control. Behaviour Research and Therapy, 51(6), 290-299. doi:10.1016/j.brat.2013.02.006

Verbeken, S., Braet, C., Lammertyn, J., Goossens, L., \& Moens, E. (2012). How is reward sensitivity related to bodyweight in children? Appetite, 58(2), 478-483.

Vervoort, L., Vandeweghe, L., Vandewalle, J., Van Durme, K., Vandevivere, E., Wante, L., et al. (2015). Measuring Punishment and Reward Sensitivity in children and adolescents with a parent-report version of the Bis/Bas-scales. Personality and Individual Differences, 87, 272277. doi:http://dx.doi.org/10.1016/j.paid.2015.08.024

Voigt, D. C., Dillard, J. P., Braddock, K. H., Anderson, J. W., Sopory, P., \& Stephenson, M. T. (2009). Carver and White's (1994) BIS/BAS scales and their relationship to risky health behaviours. Personality and Individual Differences, 47(2), 89-93. doi:10.1016/j.paid.2009.02.003

Wang, Y., \& Lim, H. (2012). The global childhood obesity epidemic and the association between socio-economic status and childhood obesity. International Review of Psychiatry, 24(3), 176-188. 
569 Table 1. Food indices based on the food categories included in the Children's Eating Habits Questionnaire - Food

570 Frequency Questionnaire

Fast food consumption frequency
$=$ Weekly consumption frequency of the
following food categories

Fried potatoes, potato croquettes

Pizza as main dish

Chips, tortillas, popcorn

Sausage roll, cheese roll, pizza-snack

Hamburger, hotdog, kebab, wrap, pita, durum

Ketchup

Mayonnaise, mayonnaise based products

\begin{tabular}{l}
\hline \multicolumn{1}{c}{ Sweet food consumption frequency } \\
\hline Candies, marshmallow \\
Chocolate, candy bars \\
Biscuits, cakes, pastries \\
Ice cream \\
\hline Sweet drink consumption frequency \\
Fruit juice \\
Sweet and soft drinks \\
Light and zero soft drinks \\
Sugared milk
\end{tabular}

573 Table 2. Descriptive data of the key variables

\begin{tabular}{lcccccccc}
\hline & $\mathrm{N}$ & Min & P25 & P50 & \multicolumn{1}{c}{ P75 } & Max & \multicolumn{1}{c}{ M } & sd \\
\hline Age (years) & 443 & 5.65 & 7.80 & 8.98 & 10.07 & 11.95 & 8.86 & 1.48 \\
RS (range 4-16) & 443 & 4.00 & 6.00 & 8.00 & 11.00 & 16.00 & 8.66 & 2.84 \\
Fast food CF (times per week) & 427 & 0.00 & 2.00 & 6.00 & 8.00 & 20.00 & 6.09 & 4.26 \\
Sweet food CF (times per week) & 431 & 0.00 & 5.00 & 9.00 & 13.00 & 30.00 & 9.25 & 5.63 \\
Sweet drink CF (times per week) & 427 & 0.00 & 4.00 & 8.00 & 14.00 & 37.00 & 9.32 & 6.94 \\
Screen time (hours per week) & 383 & 0.50 & 6.75 & 9.50 & 15.25 & 33.00 & 11.06 & 6.07 \\
PAclub (hours per week) & 387 & 0.00 & 1.00 & 2.00 & 3.50 & 8.00 & 2.29 & 1.68 \\
zBMI & 443 & -2.89 & -0.83 & -0.22 & 0.34 & 2.19 & -0.22 & 0.91 \\
\hline
\end{tabular}

574 N, number. Min, minimum. P25, percentile 25. P50, median. P75, percentile 75. Max, maximum. M, mean. sd, standard 575 deviation. RS, reward sensitivity. CF, weekly consumption frequency. PAclub, physical activity in sports clubs. zBMI, 576 age- and sex-adjusted z-score of Body Mass Index. 
Table 3. Correlations between reward sensitivity, age and weight-related behaviors and comparisons of these variables across sex and parental education level

\begin{tabular}{|c|c|c|c|c|c|c|c|c|c|c|c|c|c|c|}
\hline & \multicolumn{2}{|l|}{$\mathrm{RS}$} & \multicolumn{2}{|l|}{ Age } & \multicolumn{2}{|c|}{ Fast food $\mathrm{CF}^{\mathrm{a}}$} & \multicolumn{2}{|c|}{ Sweet food $\mathrm{CF}^{\mathrm{a}}$} & \multicolumn{2}{|c|}{ Sweet drink $\mathrm{CF}^{\mathrm{a}}$} & \multicolumn{2}{|c|}{ Screen time } & \multicolumn{2}{|l|}{ PAclub } \\
\hline & $r$ & $P$ & $\mathrm{r}$ & $P$ & $\mathrm{r}$ & $P$ & $\mathrm{r}$ & $P$ & $\mathrm{r}$ & $P$ & $\mathrm{r}$ & $P$ & $\mathrm{r}$ & $P$ \\
\hline $\operatorname{Age}^{\mathrm{b}}$ & -0.09 & 0.06 & & & & & & & & & & & & \\
\hline Fast food $\mathrm{CF}^{\mathrm{a}, \mathrm{b}}$ & 0.09 & 0.06 & 0.08 & 0.10 & & & & & & & & & & \\
\hline Sweet food $\mathrm{CF}^{\mathrm{a}, \mathrm{b}}$ & -0.03 & 0.51 & -0.09 & 0.07 & $0.10^{*}$ & 0.05 & & & & & & & & \\
\hline Sweet drink $\mathrm{CF}^{\mathrm{a}, \mathrm{b}}$ & 0.09 & 0.06 & 0.03 & 0.57 & $0.26 * *$ & $<0.01$ & $0.15 * *$ & $<0.01$ & & & & & & \\
\hline Screen time $\mathrm{e}^{\mathrm{b}}$ & 0.03 & 0.62 & $0.23 * *$ & $<0.01$ & $0.29 * *$ & $<0.01$ & $0.11^{*}$ & 0.04 & $0.14 * *$ & 0.01 & & & & \\
\hline \multirow[t]{2}{*}{ PAclub $^{c}$} & 0.02 & 0.67 & $0.16^{* *}$ & $<0.01$ & $<-0.01$ & 0.96 & $<-0.01$ & 0.87 & -0.03 & 0.55 & 0.03 & 0.63 & & \\
\hline & $t(d f)$ & $P$ & $t(d f)$ & $P$ & $t(d f)$ & $P$ & $t(d f)$ & $P$ & $\mathrm{t}(\mathrm{df})$ & $P$ & $t(d f)$ & $P$ & $\mathrm{U}(\mathrm{Z})$ & $P$ \\
\hline Sex & $1.82(441)$ & 0.07 & $-0.35(441)$ & 0.73 & $0.59(425)$ & 0.56 & $0.54(429)$ & 0.59 & $0.82(425)$ & 0.41 & $1.76(359.44)$ & 0.08 & $16604.00(-1.61)$ & 0.11 \\
\hline PEL & $0.47(411)$ & 0.64 & $1.66(411)$ & 0.10 & $3.99(396) * *$ & $<0.01$ & $-1.24(400)$ & 0.22 & $3.60(395)^{* *}$ & $<0.01$ & $3.15(367)^{* *}$ & $<0.01$ & $11294.00(-1.07)$ & 0.29 \\
\hline
\end{tabular}

RS, reward sensitivity. CF, weekly consumption frequency. PAclub, physical activity in sports clubs. r, correlation coefficient. $t(d f)$, t-value and degrees of freedom of unpaired t-

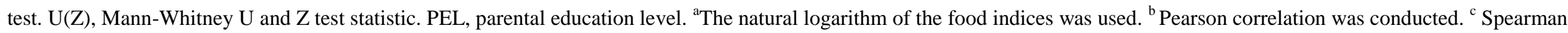
correlation was conducted. $* P<0.05, * * P<0.01$.

Table 4. Linear and logistic regressions with reward sensitivity as predictor and weight-related behaviors as dependent variables

\begin{tabular}{|c|c|c|c|c|c|c|c|c|c|c|c|c|c|c|}
\hline \multirow[t]{2}{*}{ Dependent variable } & \multicolumn{3}{|c|}{ Intercept } & \multicolumn{3}{|c|}{ RS } & \multicolumn{3}{|c|}{$\mathrm{Age}^{\mathrm{b}}$} & \multicolumn{3}{|c|}{$\operatorname{Sex}^{\mathrm{c}}$} & \multirow[b]{2}{*}{$\mathrm{r}_{\mathrm{sp}}(\mathrm{RS})$} & \multirow[b]{2}{*}{$\mathrm{R}^{2}$} \\
\hline & $\mathrm{N}$ & $\mathrm{b}(\mathrm{SE})$ & $P$ & $\mathrm{~b}(\mathrm{SE})$ & $\beta$ & $P$ & $\mathrm{~b}(\mathrm{SE})$ & $\beta$ & $P$ & $\mathrm{~b}(\mathrm{SE})$ & $\beta$ & $P$ & & \\
\hline Fast food $\mathrm{CF}^{\mathrm{a}}$ & 427 & $2.42(0.05)$ & $<0.01$ & $0.01(0.01)$ & 0.10 & 0.04 & $0.02(0.01)$ & 0.09 & 0.07 & & & & 0.10 & 0.02 \\
\hline Sweet food $\mathrm{CF}^{\mathrm{a}}$ & 431 & $2.55(0.07)$ & $<0.01$ & $-0.01(0.01)$ & -0.04 & 0.42 & $-0.03(0.01)$ & -0.09 & 0.06 & & & & -0.04 & 0.01 \\
\hline Sweet drink $\mathrm{CF}^{\mathrm{a}}$ & 427 & $2.30(0.08)$ & $<0.01$ & $0.02(0.01)$ & 0.10 & 0.05 & $0.01(0.02)$ & 0.04 & 0.46 & & & & 0.10 & 0.01 \\
\hline \multirow[t]{2}{*}{ Screen time } & 383 & $10.92(1.04)$ & $<0.01$ & $0.08(0.11)$ & 0.04 & 0.44 & $0.96(0.20)$ & 0.24 & $<0.01$ & $-1.13(0.61)$ & -0.09 & 0.10 & 0.04 & 0.06 \\
\hline & $\mathrm{N}$ & $\mathrm{b}_{\log }(\mathrm{SE})$ & $P$ & $\mathrm{~b}_{\log }(\mathrm{SE})$ & OR & $P$ & $b_{\log }(S E)$ & OR & $P$ & Nagelkerke R2 & & & & \\
\hline
\end{tabular}

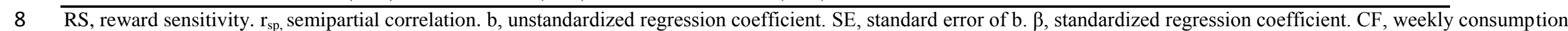

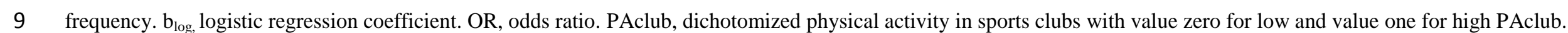

$10{ }^{\mathrm{a}}$ The natural logarithm of the food indices was used. ${ }^{\mathrm{b}}$ Centralized child age. ${ }^{\mathrm{c}}$ Value zero for boys, value one for girls. 
1 Table 5. Linear regression with Body Mass Index as dependent variable. Weight-related behaviors and parental

2 education level were included as predictors in step 1, reward sensitivity was added as predictor in step 2.

\begin{tabular}{|c|c|c|c|c|c|}
\hline \multicolumn{6}{|c|}{$\begin{array}{l}\text { Dependent variable: } \\
\text { zBMI }\end{array}$} \\
\hline & Predictor & $b$ & SE & $\beta$ & $P$ \\
\hline \multirow{7}{*}{$\begin{array}{l}\text { Step 1 } \\
\mathrm{R}^{2}=0.08\end{array}$} & Intercept & 0.35 & 0.19 & & 0.06 \\
\hline & $\begin{array}{l}\text { Fast food } \\
\text { CF }\end{array}$ & 0.02 & 0.01 & 0.11 & 0.06 \\
\hline & $\begin{array}{l}\text { Sweet } \\
\text { food } \mathrm{CF}\end{array}$ & -0.03 & 0.01 & -0.15 & $<0.01$ \\
\hline & $\begin{array}{l}\text { Sweet } \\
\text { drink CF }\end{array}$ & $<0.01$ & 0.01 & -0.03 & 0.53 \\
\hline & $\begin{array}{l}\text { Screen } \\
\text { time }\end{array}$ & $<0.01$ & 0.01 & -0.02 & 0.73 \\
\hline & PAclub & -0.04 & 0.03 & -0.07 & 0.19 \\
\hline & PEL & -0.39 & 0.12 & -0.18 & $<0.01$ \\
\hline \multirow{8}{*}{$\begin{array}{l}\text { Step } 2 \\
\mathrm{R}^{2}=0.10 \\
P\left(\Delta \mathrm{R}^{2}\right)<0.01\end{array}$} & Intercept & -0.04 & 0.23 & & 0.85 \\
\hline & $\begin{array}{l}\text { Fast food } \\
\text { CF }\end{array}$ & 0.02 & 0.01 & 0.09 & 0.09 \\
\hline & $\begin{array}{l}\text { Sweet } \\
\text { food } \mathrm{CF}\end{array}$ & -0.02 & 0.01 & -0.15 & 0.01 \\
\hline & $\begin{array}{l}\text { Sweet } \\
\text { drink CF }\end{array}$ & -0.01 & 0.01 & -0.05 & 0.4 \\
\hline & $\begin{array}{l}\text { Screen } \\
\text { time }\end{array}$ & $<0.01$ & 0.01 & -0.02 & 0.74 \\
\hline & PAclub & -0.04 & 0.03 & -0.07 & 0.15 \\
\hline & PEL & -0.39 & 0.12 & -0.18 & $<0.01$ \\
\hline & $\mathrm{RS}^{\mathrm{a}}$ & 0.05 & 0.02 & 0.15 & $<0.01$ \\
\hline
\end{tabular}

3 zBMI, age- and sex-adjusted z-score of Body Mass Index. b, unstandardized regression coefficient. SE, standard error

4 of b. $\beta$, standardized regression coefficient. $\mathrm{CF}$, weekly consumption frequency. PAclub, physical activity in sports

5 clubs. PEL, parental education level, value zero for low and value one for high PEL. $P\left(\Delta \mathrm{R}^{2}\right), P$ value of the $\mathrm{R}^{2}$ change

6 between step 1 and step 2. RS, reward sensitivity. ${ }^{\text {a }}$ Semi-partial correlation of RS is 0.15 . 